\title{
Multimodal Biometric System: A Feature Level Fusion Approach
}

\author{
K.Geetha \\ Associate Professor/Dept of IT \\ MIET Engineering College,Trichy-7
}

\author{
V.Radhakrishnan, Ph.D \\ Dean,Academic \\ Mookambigai College of Engineering,Pudukkottai
}

\begin{abstract}
Biometric systems are automatic process to identify a person through physical traits or to verify his/her identity. Various systems were implemented and used over years, and they include systems based on fingerprints, irises, facial images, hand geometry, and speaker recognition. For successful implementation of biometric systems, it is required to address many issues like accuracy, efficiency, robustness, applicability, and universality. Single modality based recognition verification $\mathrm{s}$ not very robust while combining information from various biometric modalities provides better performance. The multimodal biometric system uses multiple biometrics and integrates information for identification. It compensatesthe limitations of unimodal biometric systems. In this paper, a Multimodal biometric system proposed based on fingerprint and palmprint. Coiflet wavelets are used to extract features from the fingerprint and palmprint. It is proposed to fuse the extracted features and these features are classified using Support Vector Machine (SVM).
\end{abstract}

\section{Keywords}

Biometric, Multimodal, Unimodal, Feature Level Fusion, Coiflet wavelets, Support Vector Machine (SVM)

\section{INTRODUCTION}

Biometric technologies are automated means to verify/recognize a living person's identity based on physiological/behavioural characteristics [1]. Biometrics use characteristics which are physical like fingerprint,hand shape, facial characteristics, voice, or iris. Biometrics can also use learned/acquired characteristics including behavioral traits like signature, speech [2]. Based on application, a biometric system operates both in verification and identification modes:

In Verification mode,the system confirms a person's identity by comparing captured biometric data with biometric template in a system database. During verification, a person claims his/her identity through a Personal Identification Number (PIN), a user name or smart card. The biometric system does a one-to-one comparison and decides whether the claim is true.

In Identification mode, the system recognizes persons by searching templates of database users for a correct match. The biometric system carries out a one-to-many comparison to establish individual identity without the individual claiming an identity [3].

Biometric authentication/verification systems are usually pattern recognition systems comprising of 4 modules [4]: (i) data acquisition module, which captures an individual's biometric sample, e.g., fingerprint image, palmprint, or face; (ii) feature extraction module, where a set of representative features are extracted from acquired biometric sample; (iii) matching and decision making module that compares computed feature set with a template, (which contains the extracted feature sets during enrolment), and putting out a similarity score which decides the validity of identity claimed by an individual (iv) the system's database module, used by verification systems to store enrolled users templates.

Unimodal biometric systems recognise persons based on single biometric information. The performance of Unimodal system is affected by noisy sensor data and non-universality. Lack of invariant representation leads to un-identical of data of user biometric data during verification and the generated user's template during enrolment [5]. Some problems affecting unimodal biometric systems can be offset by multimodal biometric systems as such system consolidatesinformation obtained from two or more biometric sources for person recognition and hence they are called multimodal biometric systems.

Multimodal biometric systems have many advantages over unimodal systems, as combining evidence from various modalities through fusion greatly improves overall system accuracy. A multimodal system reduces failure to enrol and resists spoofing as it is difficult to spoof multiple biometric sources simultaneously. Multimodal systems can search large databases efficiently and quickly through use of simple but less accurate modality to narrow down the options in the database before applying complex and accurate modality on remaining data for final identification. The disadvantages of multimodal systems are that it is expensive and necessity additional resources for computation and storage when compared to unimodal systems. Multimodal systems also require more time for enrolment/verification inconveniencing users. Lastly, system accuracy actually degrades when compared to unimodal systems if proper techniques are not followed when combining evidence from various modalities. But their advantages outweigh their limitations and hence they are increasingly deployed in security related applications $[6$, 7].

Multimodal systems collect more data from subjects. Data is either fused or data processing results are fused for authentication. Biometric fusion is defined as use of multiple types of biometric data/processing methods to improve system performance. Combining modalities assures better robustness and provides a measure of adaptability to circumstances. May approaches can be adopted to combine different modalities, but two major approaches are feature fusion and decision fusion which are also known as early and late fusion [8].

A basic approach to decision fusion is treating two modalities independently. For example, face verification is performed first followed by iris verification if the former was successful. Identification is positive when the latter too is successful. Alternatively in feature fusion, feature vectors from samples jointly train a combined classifier whose advantage is that all feature information is present in the classification stage itself. 
The issue of efficient combining of biometric modalities continues to attract attention.

Feature fusion of palmprint and fingerprint features forms the basis of the multimodal system in this study. Fingerprint verification is a very reliable biometric authentication process due to its universality, permanence,distinctiveness, and accuracy. Current fingerprint recognition algorithms are based on matching minutiae features which represent local deviation in hand ridge flow. The fingerprint template stores all minutiae locations, orientations and structural relationship among points detected during enrolment [9]. During identification, deformation between two point sets is obtained and a similarity score is applied to such points.

Palm print is a relatively new physiological biometric due to its stability and unique characteristics. Palm print contain rich information with distinctive features like principal lines, wrinkles, ridges and valleys on palm surface and unlike fingerprint identification that matches one feature to many in a database, palmprint verification process matches one-to-one $[10,11] . I n$ the proposed Multimodal biometric system, fingerprint and palmprint are the modalities used. Coiflet wavelets are used to extract features from the fingerprint and palmprint. It is proposed to fuse the extracted features and these features are classified using Support Vector Machine (SVM).

\section{RELATED WORKS}

$\mathrm{Xu}$ et al [12] proposed a novel image-based linear discriminant analysis (IBLDA) to fuse two biometric traits of the same subject in a matrix at the feature level. IBLDA initially integrates a subject's two biometric traits into a complex matrix and directly extracts low-dimensional features for integrated biometric traits. IBLDA enables more information to be exploited than matching score level fusion and decision level fusion. Compared to linear discriminant analysis (LDA), IBLDA has many advantages: First, it overcomes small sample size problem that conventional LDA usually is unable to handle Second, IBLDA solves the eigen equation at a low computational cost. Third, when storing scatter matrices IBLDA does not bring a heavy memory burden as conventional LDA. Experiments show that the proposed method obtains high classification accuracy.

Dass et al [13] proposed an optimal framework to combine matching scores from multiple modalities using a likelihood ratio statistic computed through use of generalized densities estimated from genuine and impostor matching scores. The motivation to sue generalized densities is that some portions of score distributions are discrete in nature; hence, estimating distribution using continuous densities could be inappropriate. Two approaches were presented to combine evidence based on generalized densities: (i) product rule, which assumes independence between individual modalities, and (ii) copula models, that consider dependence between matching scores of multiple modalities. Experiments on MSU and NIST multimodal databases reveal that both fusion rules achieve consistently high performance.

Monwar et al [14] proposed an effective fusion scheme combining information from multiple domain experts based on rank-level fusion integration. The developed multimodal biometric system possesses many special qualities, starting from utilizing principal component analysis and Fisher's linear discriminant methods for individual matchers (face, ear, and signature) identity authentication and utilizing the novel ranklevel fusion to consolidate results from various biometric matchers. Ranks of individual matchers are combined using through use of highest rank, Borda count, and logistic regression approaches. Results show that fusion of individual modalities does improve biometric system's overall performance even when low quality data is present.

Ross et al [15] discussed fusion at the feature level as follows: (i) For face - fusion of PCA and LDA co-efficient; and fusion of LDA coefficients corresponding to the R, G, B channels; (ii) feature level fusion of face and hand modalities. Initial results are satisfactory and help emphasise the pros and cons of fusion at this level. The motivation of this work is to show the viability of such a fusion and to emphasise the need to pursue further research.

Conti et al [16] presented a template-level fusion algorithm based on iris and fingerprint traits which worked on a unified biometric descriptor for an innovative multimodal biometric identification system. A frequency-based codifying approach led to a homogenous vector of fingerprint and iris information. Successively, the Hamming Distance (HD) between two vectors obtains a similarity degree. To evaluate and compare the proposed approach's effectiveness, different tests on the official fingerprint verification competition (FVC) 2002 DB2 fingerprint database and the University of Bath Iris Image Database (BATH) iris database were performed.

\section{METHODOLOGY}

Figure 1 illustrates a typical multimodal biometric authentication system having three main steps: preprocessing, feature extraction and fusion [17]. 1. Input Biometric image (palmprint and finger print in this study). 2. For palmprint - features like line, texture is extracted and line and minutiae features for fingerprint. 3. Features are fused (wavelet data fusion) and feature set is reduced. 4. Image classified based on Nearest neighbourhood algorithm with distance calculation. 5. On classification, and matching score computation, the matched image given as output.

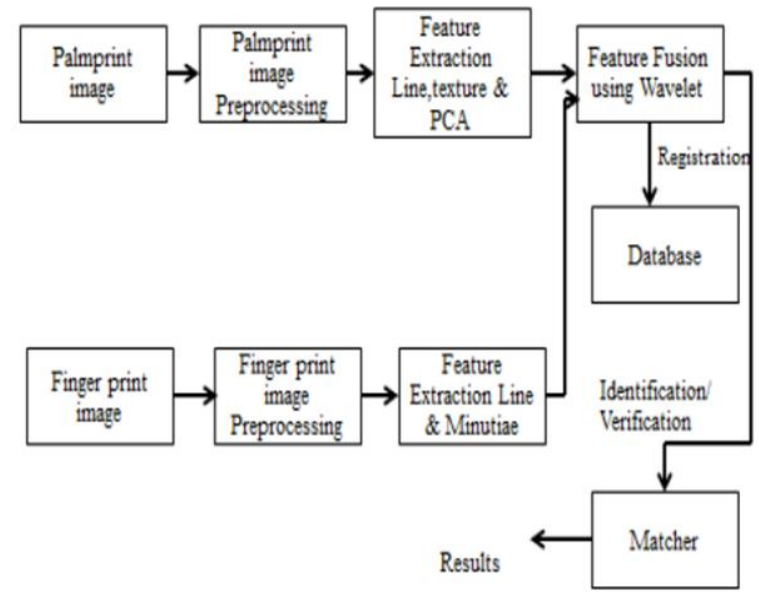

Figure 1: Illustration of multimodal biometric system

\subsection{Feature CoifletWavelet \\ Extraction using}

Coiflets are discrete wavelets designed by Daubechies [18], having scaling functions with vanishing moments. It has N/3 vanishing moments and $\mathrm{N} / 3-1$ scaling functionsand near symmetric. The function $\Psi$ has $2 \mathrm{~N}$ moments equal to 0 and the function $\varphi$ has $2 \mathrm{~N}-1$ moments equal to 0 . The two functions have a support of length $6 \mathrm{~N}-1$ [19]. 
The coifN $\Psi$ and $\varphi$ are much more symmetrical than the $d b N s$. Regarding the support length, coifN has to be compared to $d b 3 N$ or sym $3 N$. Regarding the number of vanishing moments of $\Psi$, coifN has to be compared to $d b 2 N$ or $s y m 2 N$.

If $s$ is a sufficiently regular continuous time signal, for large $j$ the coefficient

$\left\langle s, \phi_{j, k}\right\rangle$ is approximated by $2^{-j / 2} s\left(2^{-j} k\right)$

If $\mathrm{s}$ is a polynomial of degree $\mathrm{d}, \mathrm{d} \leq \mathrm{N}-1$, then the approximation becomes an equality.

\subsection{Feature fusion of palmprint and fingerprint}

In feature-level fusion, feature sets from multiple biometric algorithms are combined into a single feature set. The primary benefit of feature- level fusion is detection of correlated feature values generated by different biometric algorithms and, also identifying a salient features set that improves recognition accuracy. Eliciting this feature set requires use of dimensionality reduction methods and, hence, feature-level fusion assumes that a large number of training data is available. Also, fused feature sets are expected to reside in commensurate vector space to permit application of a suitable matching technique when feature sets [20] are consolidated. This paper proposes a new technique in biometric authentication through fusing palmprint and fingerprint with an efficient dimensionality reduction after feature fusion.

Feature extraction uses 2D Gabor filter; stationary wavelet transform and principal component analysis to extract the texture, line and appearance based features respectively. These are concatenated for feature fusion, but dimensions of fused feature template become large and so are difficult to match score computations. To offset this; a new wavelet based feature fusion technique that reduces feature template dimensionality is proposed.

Due to the spatial and frequency localizationof Gabor filters, it is used for texture segmentation. Mathematically, it is given by

$$
\begin{aligned}
& G(x ; y)=g(x ; y) * \exp (2 \pi i f(x \cos (\theta)+y \sin (\theta))) \\
& \text { Here } g(x ; y)=\left(1 / 2 \pi \sigma^{2}\right) * \exp \left(-\left(x^{2}+y^{2}\right) / 2 \sigma^{2}\right)
\end{aligned}
$$

f - frequency

$\theta$-orientation of the sinusoidal signal andlies in the interval $\left[0^{\circ}-360^{\circ}\right]$

$\mathrm{g}(\mathrm{x} ; \mathrm{y})$ - Gaussian function with scale parameter $\sigma ; \mathrm{f} ; \theta$;

$\sigma$-parameter space of Gabor filters

Theoretically, a Gabor filter is determined by the parameters F; $\theta ; \sigma$ [21]. By carefully selecting these three parameter values an optimal Gabor filter is designed.

\subsection{Algorithm}

(1) Assign value of $F$.

(2) Input image converted into a 2D matrix.

(3) Compute filter impulse response as follows:

$$
G(x ; y)=g(x ; y) * \exp (2 \pi i f(x \cos (\theta)+y \sin (\theta)))
$$

where

$$
g(x ; y)=\left(1 / 2 \pi \sigma^{2}\right) * \exp \left(-\left(x^{2}+y^{2}\right) / 2 \sigma^{2}\right)
$$

(4) The input image isfiltered by convolving it through the filter with the impulse response calculated in step3 with $\mathrm{r}(\mathrm{x} ; \mathrm{y})$ being the filtered output.

Line features are powerful and provide greater accuracy for palmprint and fingerprint based biometrics. Line detectorsare applied to extract principle lines and major wrinkle from the palmprint[22]. The common problems of such methods include noise effects,computational complexity due to large feature sets; difficulty in matching, androtation variance. To avoid them, a simple wavelet based edge detection method as described in [23] with modification is used in this study. Stationary wavelet transformation is used instead of discrete wavelet transformation. The horizontal line/edge information (represented in strong coefficients) are contained inhorizontal sub-band and vertical and diagonal sub-bands have vertical and diagonal line information respectively. The horizontal, vertical and diagonal (HVD) sub-bands are fused as follows:

$$
\text { Line }(x ; y)=\max (\operatorname{HLine}(x ; y) ; \operatorname{VLine}(x ; y) ; \operatorname{DLine}(x ; y))
$$

Fusion methods like mean-mean, max-min, img1, img2, mean-maxare used for merging wavelet decompositions of two original images; applied to approximation coefficients and detail coefficients. This study uses mean-max fusion method. The steps involved in feature fusion strategy are:

(1) Apply wavelet extension to obtain similar dimensionsof Gabor and line feature vectors.

(2) Perform wavelet decomposition using Coiflet wavelet transformation on both feature vectors.

(3) Mean ruleapplied to perform image fusion.

(4) Max ruleapplied to perform image fusion.

The combined feature vectors are classified using SVM.

SVM is useful for data classification. Classification usually involves training and testing data having some data instances. Each training set instance has one target value and many attributes. SVM aims to produce a model which predicts target value of data instances in the testing set which has only attributes.

When a set of features represented in space is given, SVM maps features non-linearly into $\mathrm{n}$ dimensional feature space. To avoid high computation, a kernel is introduced as the algorithm uses only the inputs of scalar products. Classification is solved by converting the problem into a convex quadratic optimization problem with a unique solution being obtained due to convexity [24]. The predictor variable is called an attribute in SVM; a feature is a transformed attribute. Vector is a set of features describing an example. Features define the hyperplane. SVM aims to locate the optimal hyperplane separating vector clusters with one class of attributes on one side of plane and the rest on the other. The distance between hyperplane and support vectors is the margin. SVM analysis so orients the margin that the margin between support vectors is maximized. 
Given a training set of $\left(x_{i}, y_{i}\right), i 1,2, \ldots . l$ where $x_{i} \in R^{n}$ and $y \in\{1,-1\}^{l}$, SVM has to solve the optimization problem [15] of:

$$
\min _{w, b, \xi} \frac{1}{2} w^{T} w+C \sum_{i=1}^{l} \xi_{i}
$$

Subject to $y_{i}\left(w^{T} \phi\left(x_{i}\right)+b\right) \geq 1-\xi_{i}$ and $\xi_{i} \geq 0$.

The function $\phi$ maps the vectors $x_{i}$ in higher dimensional space. $\mathrm{C}>0$ is penalty parameter of the error term.

\section{RESULTS AND DISCUSSION}

The work uses a palmprint database developed at the biometric research centre at Hong Kong Polytechnic University containing 8000 gray scale; low resolution (75 dpi) palmprints. The palmprint in the dataset were captured using CCD camera under a pegged environment. Each palm contains twenty samples. The samples are taken in two sessions and the average time interval between first and second sessions is two months. Fingerprints were selected from FVC2002 DB4B dataset. Sample palmprint image and fingerprint images are shown in Figure 2 and 3.
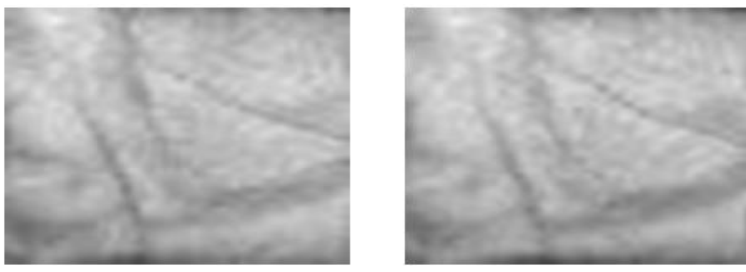

Fig. 2: Sample palmprint images
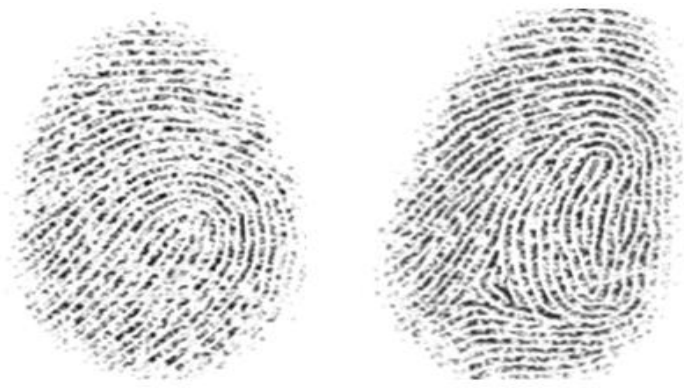

Fig. 3: Sample finger print images

Features were extracted from the palmprint and fingerprint. The features were fused and classified using Naïve Bayes and SVM with RBF kernel. Table 1 summarizes the results of the experiments.
Table 1: Classification Accuracy and RMSE

\begin{tabular}{|l|l|l|}
\hline Technique & $\begin{array}{l}\text { Classification } \\
\text { Accuracy }\end{array}$ & RMSE \\
\hline Naïve Bayes & $86.42 \%$ & 0.1228 \\
\hline SVM with RBF kernel & $97.53 \%$ & 0.0524 \\
\hline SVM with linear kernel & $96.91 \%$ & 0.0586 \\
\hline
\end{tabular}

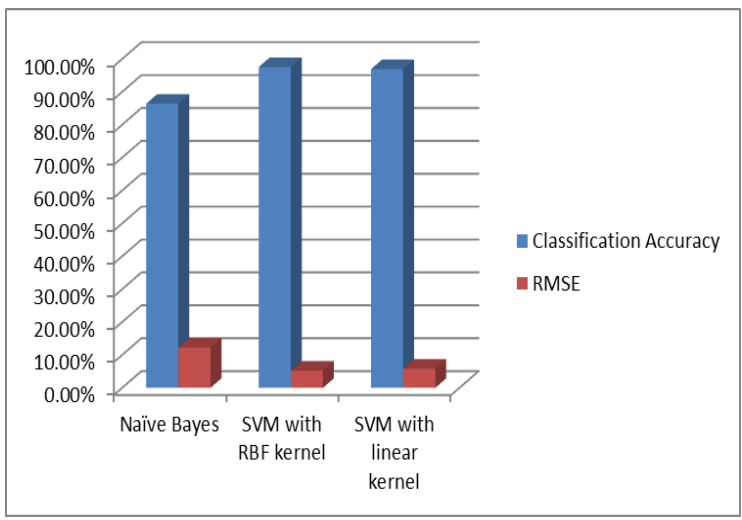

Figure 4: Classification Accuracy and RMSE

It is observed from the graph that the SVM with RBF kernel achieves the best classification of $97.53 \%$ and a low root mean square error of 0.0524 . Table 2 tabulates the Precision, Recall and $f$ Measure for different techniques.

Table 2: Precision, Recall and f Measure

\begin{tabular}{|l|l|l|l|}
\hline Technique & Precision & Recall & $\begin{array}{l}\text { F- } \\
\text { Measure }\end{array}$ \\
\hline Naïve Bayes & 0.888 & 0.864 & 0.858 \\
\hline SVM with RBF kernel & 0.981 & 0.975 & 0.976 \\
\hline SVM with linear kernel & 0.976 & 0.969 & 0.97 \\
\hline
\end{tabular}

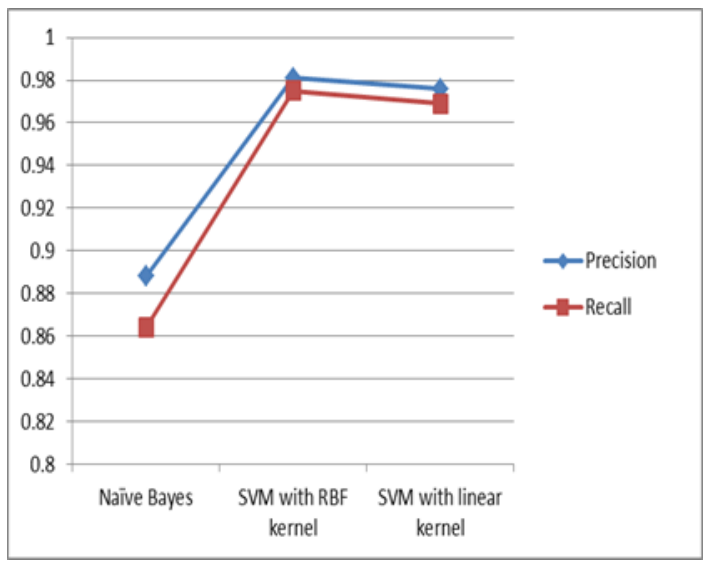

Figure 5: Precision and Recall

The precision and recall achieved by SVM with RBF kernel is high which is a requirement in authentication systems. 


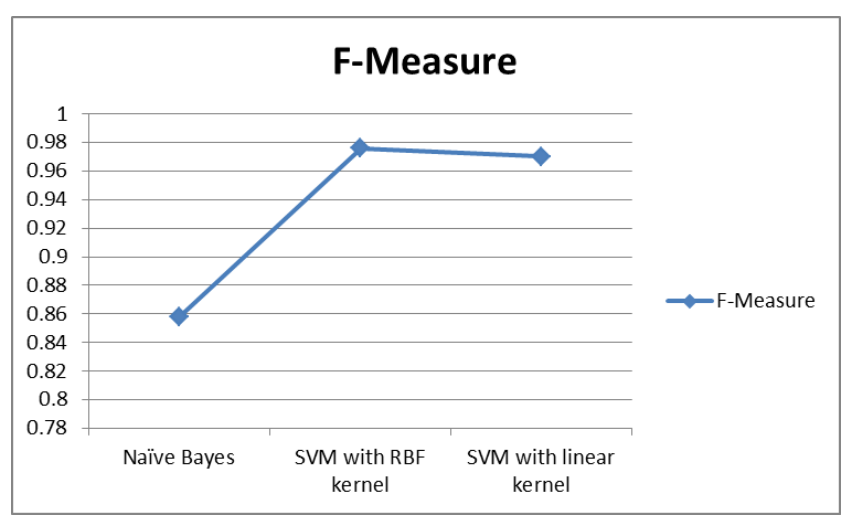

Figure 6: f Measure

\section{CONCLUSION}

Multimodal biometrics is popular currently due to its performance and advanced security level. This paper presents various issues related to multimodal biometrics systems. Combining multiple biometric traits improve system performance. In the proposed Multimodal biometric system, fingerprint and palmprint are modalities used. Coiflet wavelets extract features from fingerprint and palmprint. It is proposed to fuse extracted features and they are classified using Support Vector Machine (SVM). Experimental results reveal satisfactory classification accuracy for feature fusion and SVM.

\section{REFERENCES}

[1] Jain, A., \&Verma, C. K. (2012). A Framework Based On Hybrid Biometrics For Personal Verification Systems. International Journal of Applied, 1(1), 55-58.

[2] Wayman, J., Jain, A., Maltoni, D., \&Maio, D. (2005). An introduction to biometric authentication systems. Biometric Systems, 1-20.

[3] Fierrez-Aguilar, J., Ortega-Garcia, J., GonzalezRodriguez, J., \&Bigun, J. (2005). Discriminative multimodal biometric authentication based on quality measures. Pattern Recognition, 38(5), 777-779.

[4] Jain, A.K., Ross, A., and Prabhakar, S.: An Introduction to Biometric Recognition. IEEE Transactions on Circuits and Systems for Video Technology, 14 (2004), 4-20.

[5] Ross, A., \& Jain, A. K. (2004, September). Multimodal biometrics: An overview. In Proceedings of 12th European Signal Processing Conference (pp. 12211224).

[6] Snelick, R., Uludag, U., Mink, A., Indovina, M., \& Jain, A. (2005). Large-scale evaluation of multimodal biometric authentication using state-of-the-art systems. Pattern Analysis and Machine Intelligence, IEEE Transactions on, 27(3), 450-455.

[7] Shukla, A., Tiwari, R., \& Kala, R. (2010). Multimodal Biometric Systems. Towards Hybrid and Adaptive Computing, 401-418.
[8] Ross, A., \& Jain, A. (2003). Information fusion in biometrics. Pattern recognition letters, 24(13), 21152125.Chikkerur, S., Wu, C., \&Govindaraju, V. (2004). A systematic approach for feature extraction in fingerprint images. Biometric Authentication, 1-23.Zhang, D. D., Zhang, D., \& Zhang, D. (2004). Palmprint authentication (Vol. 1). Norwell: Kluwer Academic Publishers.

[9] Bong, D. B. L., Tingang, R. N., \& Joseph, A. (2010). Palm Print Verification System. In Proc. of the World Congress on Engineering.

[10] Xu, Y., \& Zhang, D. (2010). Represent and fuse bimodal biometric images at the feature level: complex-matrixbased fusion scheme. Optical Engineering, 49(3), 037002-037002.

[11] Dass, S., Nandakumar, K., \& Jain, A. (2005). A principled approach to score level fusion in multimodal biometric systems. In Audio-and Video-Based Biometric Person Authentication (pp. 415-434). Springer Berlin/Heidelberg.

[12] Monwar, M. M., \&Gavrilova, M. L. (2009). Multimodal biometric system using rank-level fusion approach. Systems, Man, and Cybernetics, Part B: Cybernetics, IEEE Transactions on, 39(4), 867-878.

[13] Ross, A. A., \&Govindarajan, R. (2005, March). Feature level fusion of hand and face biometrics. In Defense and Security (pp. 196-204). International Society for Optics and Photonics.

[14] Conti, V., Militello, C., Sorbello, F., \&Vitabile, S. (2010). A frequency-based approach for features fusion in fingerprint and iris multimodal biometric identification systems. Systems, Man, and Cybernetics, Part C: Applications and Reviews, IEEE Transactions on, 40(4), 384-395.

[15] Gayathri, R., \&Ramamoorthy, P. (2012). A Fingerprint and Palmprint Recognition Approach Based on Multiple Feature Extraction. European Journal of Scientific Research. ISSN, 514-526.

[16] Ingrid Daubechies, Ten Lectures on Wavelets, Society for Industrial and Applied Mathematics, 1992.

[17] G. Beylkin, R. Coifman, and V. Rokhlin (1991),Fast wavelet transforms and numerical algorithms, Comm. Pure Appl. Math., 44, pp. 141-183

[18] Jain, A., \&Aggarwal, S. (2012). Multimodal Biometric System: A Survey. reason, 1(1), 58-63.

[19] Mehrotra, R., Namuduri, K. R., \&Ranganathan, N. (1992). Gabor filter-based edge detection. Pattern Recognition, 25(12), 1479-1494.

[20] Wu, X., Zhang, D., \& Wang, K. (2006). Palm line extraction and matching for personal authentication. Systems, Man and Cybernetics, Part A: Systems and Humans, IEEE Transactions on, 36(5), 978-987.

[21] Prasad, S. M., Govindan, V. K., \&Sathidevi, P. S. (2009, January). Bimodal personal recognition using hand images. In Proceedings of the International Conference on Advances in Computing, Communication and Control (pp. 403-409). ACM 